\title{
Predicting discharge walking function with high-intensity stepping training during inpatient rehabilitation in non-ambulatory patients post-stroke
}

Christopher E Henderson ${ }^{1-3}$, Megan Fahey ${ }^{5}$, Gabi Brazg ${ }^{5}$, Jennifer L. Moore ${ }^{3,4}$, T. George Hornby $^{1-3}$

${ }^{1}$ Department of Physical Medicine and Rehabilitation, Indiana University School of Medicine, Indianapolis IN

${ }^{2}$ Rehabilitation Hospital of Indiana, Indianapolis, IN

${ }^{3}$ Institute of Knowledge Translation

${ }^{4}$ SouthEastern Norway Regional Center for Knowledge Translation in Rehabilitation

${ }^{5}$ Shirley Ryan Ability Lab, Chicago, IL

Running Title: Locomotor recovery with high-intensity training

Number of Tables: 4

Number of Figures: 2

Abstract word count: 248

Manuscript word count: 3245

Acknowledgements/Sources of Funding: Funding was provided by NIDILRR-90RT5027.

Corresponding Author:

T. George Hornby, PhD, PT

Department of Physical Medicine and Rehabilitation

Indiana University School of Medicine

Phone: 317-329-2353; Fax: 317-329-2210

E-mail: tghornby@iu.edu

This is the author's manuscript of the article published in final edited form as:

Henderson, C. E., Fahey, M., Brazg, G., Moore, J. L., \& Hornby, T. G. (2020). Predicting Discharge Walking Function With High-Intensity Stepping Training During Inpatient Rehabilitation in Nonambulatory Patients Poststroke. Archives of Physical Medicine and Rehabilitation. https://doi.org/10.1016/j.apmr.2020.10.127 


\section{Abstract}

2 Objective: This cohort investigation identified primary predictors of discharge walking function of non-ambulatory individuals post-stroke with high-intensity training (HIT) during inpatient

4 rehabilitation.

5 Design: Observational cohort investigation.

6 Setting: Inpatient rehabilitation.

7 Participants: Data were collected from 257 individuals $<6$ months post-stroke who required 8 assistance to walk at admission.

9 Intervention: Clinical physical therapy interventions attempted to maximize stepping practice at higher intensities (HIT).

Main outcome measures: Primary outcomes included the discharge level of assistance required during walking (minimal or no assistance) and attainment of specific gait speed thresholds $(0.4$ and $0.8 \mathrm{~m} / \mathrm{s}$ ) during the 10 -meter walk test (10MWT). Independent predictors were demographics, training interventions (including steps/day), baseline Berg Balance Scale (BBS) and paretic leg strength.

Results: Participants performed a median (IQR) of 1270 (533-2297) steps/day throughout inpatient rehabilitation, with significant differences between those who walked with vs without assistance at discharge. Logistic regressions indicate steps/day was a primary predictor of unassisted walking recovery; removal of steps/day resulted in primary predictors of baseline BBS and strength. Receiver operating characteristic (ROC) analyses indicate significant areas under the curve for BBS, and relatively low cut-off scores of 5.5 points at admission to walk without assistance at any speed. ROC analyses performed using 1-week outcomes indicate BBS scores of 5-17 points were needed to achieve locomotor thresholds. 
24 Conclusion: Stepping activity, BBS and paretic leg strength were primary predictors of walking outcomes in patients performing HIT, and ROC analyses indicated recovery of independent walking could be achieved in low functioning patients early post-stroke.

Key Words: exercise therapy, rehabilitation, locomotion 


\section{Abbreviations}

30 10MWT - 10-meter walk test

31 6MWT - 6-minute walk test

32 AUC - area under the curve

33 BBS - Berg Balance Scale

$34 \quad$ HIT - high intensity training

$35 \quad \mathrm{HR}$ - heart rate

36 HRmax - maximum HR

37 FIM - Functional Independence Measure

38 LoA - level of assistance

39 ROC - receiver operating characteristics

40 RPE - ratings of perceived exertion

41

42 


\section{Introduction}

Recovery of independent ambulation is often a primary goal of rehabilitation for individuals post-stroke but is limited by the impairments in strength, balance, and endurance ${ }^{1}$. Fortunately, $80-90 \%$ of individuals post-stroke ambulate at 6-months post-injury ${ }^{2}$. During the initial recovery process, about $40 \%$ of individuals walk with minimal or no assistance at 1 -month post-stroke ${ }^{3}$, and the probability of recovery of independent ambulation in those who are initially nonambulatory is substantially lower ${ }^{4}$. Previously published algorithms indicate demographics ${ }^{3-6}$ and clinical characteristics ${ }^{3,7-10}$, including age $e^{5,6}$, strength and postural deficits ${ }^{6-10}$, and the amount of walking assistance at admission ${ }^{7,11}$, can facilitate prediction of walking recovery at pre-specified time points. For example, Bland and colleagues indicated Berg Balance Scores $(\mathrm{BBS}) \leq 20$ points and total or maximum assistance for walking at admission were likely to predict gait speeds $<0.40 \mathrm{~m} / \mathrm{s}$ at discharge ${ }^{7}$. Louie et $\mathrm{al}^{8}$ also suggested BBS $>29$ at admission to inpatient rehabilitation was predictive of achieving $>0.8 \mathrm{~m} / \mathrm{s}$. Prediction of walking recovery has important clinical implications, including informing clinicians regarding an individual's recovery potential or discharge disposition ${ }^{12,13}$.

Regardless of differences in predictive algorithms described above, most models include very limited description of physical interventions provided during rehabilitation ${ }^{6,7,10,14}$. However, observational studies of standard clinical interventions indicate provision of minimal taskspecific practice ${ }^{15-18}$ at lower cardiovascular intensities ${ }^{19-22}$. Conversely, research over the past decade indicates significant contributions of the type and cardiovascular intensity of interventions on walking outcomes ${ }^{23-25}$. Specific studies have identified dose-response relationships for both the amount (steps/day) and intensities (i.e., >70\% age-predicted maximum heart rates $[\mathrm{HR}])$ ) of stepping practice on gait speed and distance in individuals $<^{3,26}$ and $>6$ 
67 months ${ }^{27}$ post-stroke (see also ${ }^{28,29}$ ). In patients early post-stroke receiving inpatient rehabilitation during which high intensity training was prioritized during clinical physical therapy sessions, steps/day was also the primary determinant of recovery of independent walking, which in turn was the primary predictor for discharge to home. ${ }^{3,14}$ Preliminary data suggest relatively low BBS scores at admission or following the first week of rehabilitation (5-10 points) may be sufficient to achieve independent ambulation at discharge ${ }^{3}$.

Few investigations have thoroughly delineated the relative contributions of demographics, clinical presentation and training activities on locomotor outcomes, particularly with highintensity training (HIT) with attempts to maximize stepping practice. The purpose of this study was to develop algorithms that facilitate prediction of walking function at discharge from inpatient rehabilitation using available demographics, clinical characteristics and training parameters when attempting to provide HIT focused on maximizing stepping activity as standard care. Based on previous findngs ${ }^{3}$, we hypothesized that stepping activity would be a primary determinant of walking outcomes, with secondary predictors of strength and balance that could be utilized to generate algorithms that may facilitate clinical implementation. 


\section{Methods}

The present study details demographics, clinical outcomes and details of rehabilitation interventions from patients $<6$ months post-stroke receiving inpatient rehabilitation on one of two primary stroke units at a single rehabilitation facility. During clinical physical therapy sessions, therapists attempted to maximize stepping activities and achieve high cardiovascular intensities as possible. Preliminary analyses of the first cohort $(n=201)$ focused on the feasibility of providing HIT during inpatient rehabilitation and the outcomes achieved in both nonambulatory and ambulatory participants ${ }^{3}$. In the present study, data from an additional patient cohort $(n=126)$ were collected and combined with the original cohort of patients who were nonambulatory upon admission. The present study focuses only on different levels of recovery in patients who could not ambulate without physical assistance upon admission $(n=257)$.

\section{Study Sample and Design}

Individuals with a diagnosis of new stroke $<6$ months prior to admission to inpatient rehabilitation were consecutively enrolled and prospective and retrospective analyses were approved by the local Institutional Review Board. Additional inclusion criteria included 18-89 years old and able to follow a one-step command. Participants were excluded if they had restrictions in lower extremity weightbearing (e.g., amputation or fracture) or additional neurologic or orthopedic disorders that limited ambulation to $<46 \mathrm{~m}(150 \mathrm{ft})$ prior to their current injury. Per local institutional review board policy, participants were also excluded if they were pregnant or HIV positive. Demographic information were extracted from medical records as possible and included: gender, age, duration poststroke upon admission to inpatient rehabilitation, lesion side, type (ischemic/hemorrhagic/unknown), distribution (classified as cortical [anterior, middle, or posterior], subcortical, subtentorial [brainstem or cerebellar], or 
multiple lesions ${ }^{30}$. The Charlson Comorbidity Index ${ }^{31}$ was also calculated, with the most recent stroke excluded.

\section{Intervention}

The primary goal of the interventions was to attempt to maximize volitional stepping practice at high cardiovascular intensities $\left(70-85 \% \mathrm{HR}_{\max }\right)$ during inpatient physical therapy sessions. The amount of stepping activity was recorded per minute from approximately 7:30 AM to 5:00 PM on weekdays throughout the length of stay using validated accelerometers worn on the paretic ankle (StepWatch 3, Modus Health LLC, Washington DC). The prescribed training paradigm has been previously detailed in controlled studies ${ }^{26,27,32}$ and in the clinical setting ${ }^{3,29}$. Briefly, interventions were performed on a treadmill, stairs, or over ground with body weight support and/or physical assistance provided only as needed to complete the task of walking, with limited consideration of kinematics. Interventions targeted aerobic training ranges $(70-85 \%$ $\mathrm{HR}_{\max }$ as measured using pulse-oximeters with forehead sensors (Rad7, Masimo Inc, Irvine, CA), with ranges decreased by $10-15$ beats/min if prescribed beta-blockers), and Borg Rating of Perceived Exertion ${ }^{33}$ (RPE; ordinal 6-20) > 13 ("somewhat hard"). The intensity and difficulty of stepping activities were progressed as able and included reduced physical assistance, increased speed, increased resistance (ankle weights or weighted vests), and progression to uneven and unstable surfaces in multiple directions. Treating therapists were educated and trained on this general stepping paradigm described above although explicit expectations regarding the types, environments, and durations of individual activities were not provided. Rather, treating physical therapists determined the types of stepping activities performed based on their clinical judgement and availability of necessary equipment and support personnel. 
Preliminary analyses suggested median (interquartile range) heart rates of 70\% (62-76\%) predicted maximum were achieved, with median RPEs of $16(15-17)^{3}$.

\section{Outcomes}

Outcomes were assessed at admission, weekly, and at discharge, and included the 6-minute walk test (6MWT), 10-meter walk test (10MWT), and Berg Balance Scale (BBS). The 6MWT was administered with the directions to "cover as much ground as possible" over a specified course on the rehabilitation unit ${ }^{34}$. Physical assistance for the 6MWT was provided as needed, with the level of physical assistance (LoA) documented using an ordinal 7-point scale similar to the FIM for walking (1=total assistance [patient performs $0-24 \%$ of task]; $2=$ maximum [2549\%]; 3=moderate [50-74\%]; 4=minimum [75-99\%]; 5=supervision; 6=modified independent; $7=$ independent; with no distance requirements ${ }^{3}$. The 10MWT was administered using the directions 'walk at your usual, comfortable pace' and physical assistance was provided as necessary ${ }^{2}$. The BBS was used to assess postural stability during sitting and standing tasks ${ }^{35}$. Manual muscle testing of the paretic lower extremity was only performed at admission using a standard 0-5 scale averaged over the dorsiflexors, plantarflexors, and hip/knee flexors and extensors. Other information extracted from the medical record included FIM scores for bed mobility (FIM-bed), toilet transfers (FIM-Toilet), and walking subscores (FIM-Walk).

\section{Statistical Analysis}

Demographics, training interventions, and clinical discharge outcomes were tabulated, with outcomes utilized only with primary measures at admission. Missing data were imputed for 6MWT/10MWT upon initial admission, with $0 \mathrm{~m}$ or $0.0 \mathrm{~m} / \mathrm{s}$ entered for distance, and total assistance (score=1) entered for LoA only if documentation of admission FIM-Walk indicated total assistance or not attempted. For week 1 and discharge outcomes, data were imputed from 
the last outcome carried forward. Primary outcomes included BBS, 10MWT, 6MWT, 6MWT LoA (admit, week 1 and discharge), with 10MWT LoA not included given the high correlation with 6MWT LoA $(r=0.86)$. Participants were characterized as non-ambulatory if $6 \mathrm{MWT}$ LoA $\leq 4$ or ambulatory if 6MWT LoA $\geq 5$.

Data were not normally distributed (Shapiro-Wilk tests) and presented using medians and IQRs. Spearman's correlation analyses determined associations between selected demographics, details of training activities, and clinical characteristics at admission with primary outcomes at discharge (adjusted $\alpha=0.00125$ with 40 comparisons). Conditional logistic regression was also performed to evaluate contributions of independent predictors to discharge walking function dichotomized by LoA $\geq 5$ vs LoA $\leq 4$ at discharge. For these analyses, Spearman's correlations were used to test for multicollinearity between independent variables (rho $>0.70$ ), in which case parallel models were created ${ }^{8}$. In the present analyses, the strengths of correlations between all primary outcomes with other assessments at admission were similar, and BBS was chosen due to the potentially greater sensitivity to change throughout the 56-point scale and its relative contributions as detailed in other studies ${ }^{7,8}$. The resultant logistic regressions were subsequently calibrated by plotting observed vs predicted recovery of independent ambulation. Receiver operating characteristic (ROC) analysis was performed using the primary predictors identified from the logistic regression at the $1^{\text {st }}$ and $2^{\text {nd }}$ week of admission. The area under the curve (AUC) was identified, as well as the cut-off scores from the highest combined sensitivity and specificity that best distinguished the ability to reach specific threshold of walking independence and gait speed, including $\operatorname{LoA} \geq 4, \operatorname{LoA} \geq 5$ at $>0 \mathrm{~m} / \mathrm{s}, \geq 0.4 \mathrm{~m} / \mathrm{s}$, and $\geq 0.8 \mathrm{~m} / \mathrm{s}$. All analyses were performed using SPSS v26. 


\section{Results}

Retrospective data analyses on demographics, clinical characteristics and training interventions were performed on 327 patients $<6$ months post-stroke receiving inpatient rehabilitation. The specific analyses in this study focused on 257 patients who were nonambulatory at admission (i.e., requiring assistance to walk or $\operatorname{LoA} \leq 4$ ). Table 1 provides demographic characteristics and admission outcomes for the entire population, and separated by those who remained non-ambulatory at discharge $(\operatorname{LoA} \leq 4)$ or those who became ambulatory without assistance (e.g. LoA $\geq 5$ ). Approximately $79 \%$ of patients were admitted $\leq 30$ days poststroke, with most $<65$ years old $(58 \%)$ and half $(51 \%)$ with inpatient lengths of stay $\leq 30$ days. Those who required assistance at discharge $(\operatorname{LoA} \leq 4)$ were admitted to rehabilitation significantly later than those who attained $\mathrm{LoA} \geq 5$ (17 vs 12 days, respectively) and presented with lower strength scores, BBS, and FIM transfer scores (Table 1).

Table 1 and Figure 1 also detail the therapy interventions, including a median of 33 sessions over 30 days. There were no differences in total PT sessions between individuals with LoA $\leq 4$ vs LoA $\geq 5$ at discharge, although there were significant differences in lengths of stay (longer for LoA $\leq 4$ ) and sessions/day (favoring LoA $\geq 5$ ). Median steps/day across all individuals was 1270 (553-2297), with large differences between those who were $\operatorname{LoA} \leq 4$ vs $\operatorname{LoA} \geq 5$ Figure 1 delineates steps/day throughout the length of stay in individuals delineated by LoA at discharge or reaching specific walking speeds.

Primary outcomes at admission to inpatient rehabilitation, week 1 and discharge are provided for the entire population and separately for those $\operatorname{LoA} \leq 4$ and $\operatorname{LoA} \geq 5$ at discharge (Table 2). Significant differences in outcomes were observed between separated groups at every measure, with much larger differences at week 1 and discharge. Correlation analyses revealed that steps/day throughout the length of stay demonstrated the strongest correlation to BBS and 
walking outcomes (10MWT, 6MWT and LoA; Table 3). In contrast, admission BBS or walking scores demonstrated lower but significant correlations with discharge outcomes (range 0.460.61). Conditional logistic regression analyses also indicate steps/day as the primary determinant of $\mathrm{LoA} \geq 5$ at discharge at admission, and as a secondary determinant at week 1 (Equations 1-2; Supplementary Table 1).

Eq 1: $\ln \left(\frac{\mathrm{p}}{1-\mathrm{p}}\right)=0.97 * \frac{\text { steps }}{1000 * \text { day }}+0.26^{*}$ strength $+0.06^{*}$ admit BBS -1.92 ; Eq 2: $\ln \left(\frac{\mathrm{p}}{1-\mathrm{p}}\right)=0.12^{*}$ week $1 \mathrm{BBS}+0.75^{*} \frac{\text { steps }}{1000^{*} \text { day }}-2.1$;

Given the lack of equipment in many other clinical settings to monitoring stepping activity, steps/day was removed as an independent predictor during subsequent logistic regressions. Other predictors, including admit BBS, 10MWT, 6MWT, and 6MWT LoA were all highly correlated (rho=0.67-0.82) with each other, and parallel regression models were generated revealing similar findings. However, the primary independent variable remained the BBS scores given its importance in previous studies ${ }^{7,8}$ and the greater sensitivity to change throughout the 56-point scale. Resultant analyses revealed significant predictors of admit BBS, with secondary contributions of paretic strength, duration post-stroke and age at admission (Equation 3, Fig 2A), Regressions at week 1 indicate BBS was the sole predictor of unassisted walking ability (Equation 4, Fig 2B; Supplementary Table 1).

Eq 3: $\ln \left(\frac{\mathrm{p}}{1-\mathrm{p}}\right)=0.09^{*}$ admit BBS $+0.48^{*}$ strength $-0.02 *$ duration $-0.03^{*}$ age +1.2 ; Eq 4: $\ln \left(\frac{p}{1-p}\right)=0.16^{*}$ week 1 BBS - 1.5;

Model calibration was assessed by plotting observed vs predicted recovery of ambulation, revealing very good accuracy with slopes $\sim 1.0$ and strong associations $\left(\mathrm{r}^{2}=0.92\right.$ for Equation 3, 
$r^{2}=0.94$ for Equation 4). The data utilized are provided in Supplementary Table 2 with predicted vs observed plots depicted in the Supplementary Figure.

To facilitate clinical implementation, regression models were simplified by limiting independent predictors to clinical measures of strength and BBS and coefficients were adjusted to minimize calculations (please see ${ }^{36}$ as an example). For example, logistic regressions at admission revealed strength scores with nearly 3 -fold larger coefficients than BBS, and the model was simplified by using the raw values of both $3 *$ strength and BBS on the $\mathrm{x}$-axis coordinates (absolute errors between the actual vs simplified models were $<0.9 \%$; Fig $2 \mathrm{C}$ ). Similarly, week 1 BBS scores were the sole predictor of walking independently and the $\mathrm{x}$-axis was scaled accordingly (Fig 2D).

The original and simplified models are provided in Fig 2A-D. Median admission scores for paretic limb strength (0.6) and BBS (3) for those with LoA $\leq 4$ at discharge are represented, with similar data provided for a representative patient who was ambulatory at discharge (LoA $\geq 5$; median strength=2.5; $\mathrm{BBS}=6$ ). As depicted in Fig2A and $\mathrm{C}$, the representative non-ambulatory patient at discharge $(\mathrm{LoA} \leq 4)$ revealed a $38 \%$ probability of recovery of independent walking, whereas a representative patient who was ambulatory at discharge (LoA $\geq 5)$ had a $68 \%$ probability of unassisted walking. For week 1, median BBS scores for those who were nonambulatory $(\operatorname{LoA} \leq 4)$ at discharge was 4 , indicating a $31 \%$ probability of independent walking. For those who were ambulatory $(\mathrm{LoA} \geq 5)$ at discharge (median week $1 \mathrm{BBS}=20)$, the probability was79\% (Fig 2B and D).

Additional conditional regression analyses were performed for specific thresholds of gait performance ( $\operatorname{LoA} \geq 4, \operatorname{LoA} \geq 5, \geq 0.4 \mathrm{~m} / \mathrm{s}, \geq 0.8 \mathrm{~m} / \mathrm{s}$ ) with equations provided in Supplemental Table 3. BBS and paretic strength were the primary determinants at admission, with variable 
contributions from age, duration post-stroke and other variables. For week 1 scores, BBS was the primary predictor for all thresholds of locomotor recovery.

ROC analyses were performed to estimate the ability of demographic and clinical outcomes to predict locomotor recovery at the specific thresholds described previously. Table 4 details specific cut-off values and significant areas under the curve (AUCs) for age, duration poststroke, strength and primary clinical outcomes at admission and week 1. The AUCs for all primary outcomes and strength were significantly greater than age and duration post-stroke. Admit BBS $>5$ appeared to best differentiate those who would reach $\operatorname{LoA} \geq 5$ at any speed, with slightly lower values for those able to achieve $\operatorname{LoA} \geq 4$ (BBS>3). For walking outcomes, admission LoA $>2$ best differentiated recovery at all levels, with very low cut-off values for walking function needed to achieve $\mathrm{LoA} \geq 4$ (10MWT:0.01 m/s, 6MWT:2 m) or LoA $\geq 5$ (10MWT:0.03 m/s, 6MWT:6 m). At week 1, however, AUCs were significantly greater than admit AUCs for corresponding outcomes, with greater walking ability required to achieve the specific levels of walking function. Notably, week 1 cut-offs for 6MWT LoA ranged from >2-3 indicating moderate to minimal physical assistance, and BBS cut-off scores ranged from $>5$ and $>16$, respectively. 


\section{Discussion}

The current study details changes in walking and balance outcomes throughout the course of inpatient rehabilitation in individuals who were initially non-ambulatory early post-stroke $(\operatorname{LoA} \leq 4)$, and the clinical measures and cut-off scores that predict varying levels of walking recovery at discharge. With attempts to provide HIT throughout patients' length of stay, logistic analyses revealed steps/day were the primary determinant of walking function, with BBS and strength as additional primary predictors. Subsequent ROC analyses at admission and at 1 week revealed those with substantial strength and balance deficits may still recover walking function without physical assistance at discharge.

Previous studies suggest recovery of independent ambulation is a primary determinant of discharge to home following inpatient rehabilitation, and logistic and ROC analyses to predict walking outcomes following HIT in non-ambulatory individuals have not be performed. Importantly, most predictive algorithms do not detail the types and amount of physical therapy activities provided $^{7-10}$, which have been shown to influence locomotor outcomes following subacute and chronic stroke $e^{3,26,27,32}$. Consistent with preliminary analyses ${ }^{3}$, steps/day was the primary predictor of walking ability, with secondary predictors of baseline clinical assessments and selected demographics. The association between steps/day and outcomes may reflect the importance of stepping activity, or indicates those with higher function achieved greater amounts of steps. Regardless, the important finding may be is the potential influence of the amount of stepping practice provided, which was $\sim 5$-fold greater than published reports of stepping activity during traditional inpatient rehabilitation ( 250 steps/session $)^{15,18}$. These stepping amounts may have influenced the predictive algorithms of walking recovery, as demonstrated previously. 

predictors of admission assessments (BBS, 10MWT, 6MWT and LoA, and strength) as main determinants of walking recovery, . We focused primarily on the BBS, however, given the sensitivity of BBS in patients with severe motor deficits. Our findings are consistent with previous studies emphasizing the contributions of these clinical variables to walking outcomes post-stroke ${ }^{7-10}$, and approximation of these equations are provided to facilitate clinical implementation (Fig 2C-D).

The subsequent ROC analyses performed to identify predictors and cut-off scores for attainment of specific discharge levels of walking ability also allowed comparisons between different predictive algorithms from other studies. For example, a BBS $>5$ in this study predicted walking recovery without assistance (LoA $\geq 5)$ and achieving $>0.4 \mathrm{~m} / \mathrm{s}$ or $>0.8 \mathrm{~m} / \mathrm{s}$ at discharge; this value is substantially lower than previous studies to predict similar walking speed thresholds. For example, in determining which patients early post-stroke could achieve $0.4 \mathrm{~m} / \mathrm{s}$ or $0.8 \mathrm{~m} / \mathrm{s}$ at discharge from inpatient rehabilitation ${ }^{7,8}$, admission BBS $>20^{7}$ or $>29^{8}$, respectively, were found to be predictive of attaining these gait speeds. Cut-off scores for BBS at 1-week post-admission indicate BBS scores $>16$ points for $0.8 \mathrm{~m} / \mathrm{s}$, which is smaller than the scores from other studies. Differences in findings here as compared to other studies are not certain, as the sample populations described appear to be similar if not lower functioning in this present study (e.g., median BBS=5 vs mean BBS $=15,5-16^{7,8}$. As suggested above, however, differences could suggest the influence of a modifiable treatment variable, specifically prioritizing stepping practice at higher intensities, on walking recovery in those with substantial impairments.

\section{Study limitations}


Primary limitations of this study include the lack of a control group and long-term follow-up.

A recent study used a historical comparative effectiveness design to demonstrate improved magnitude of rate of recovery of locomotor outcomes following vs prior to implementation of HIT in inpatient rehabilitation ${ }^{29}$. However, that study lacked follow-up evaluations as well, and the data were collected in individuals with much higher admission walking and balance scores in a different health-care system. Performing this type of study in other settings and contexts more similar to US rehabilitation systems with long-term follow-up may provide better understanding of the comparative effectiveness of HIT during inpatient rehabilitation on longterm prognosis. An additional limitation of this study includes limited understanding of the potential influence of patient height and weight, as larger patients may be more difficult to mobilize early post-stroke. A further limitation is the lack of description of psychological factors, such as depression or patient tolerance to exercise prior to or following the stroke, which may contribute to their willingness to participate in HIT.

\section{Summary/Conclusions}

The present data suggest steps/day, BBS and strength are primary predictors of locomotor outcomes in non-ambulatory patients post-stroke when HIT that prioritizing stepping practice is provided during inpatient rehabilitation. Comparison of predictive cut-off values revealed much lower BBS scores may be needed to recover specific thresholds of walking recovery. Further research is required to assess the potential influence of HIT on long-term outcomes in patients with severe motor deficits early post-stroke, particularly as compared to more traditional interventions.

\section{Disclosures/Conflict of Interest: none}


1. Patterson SL, Forrester LW, Rodgers MM, et al. Determinants of walking function after stroke:

2. Wade DT, Wood VA, Heller A, Maggs J, Langton Hewer R. Walking after stroke. Measurement and recovery over the first 3 months. Scand J Rehabil Med. 1987;19(1):25-30.

3. Hornby TG, Holleran CL, Leddy AL, et al. Feasibility of Focused Stepping Practice During Inpatient Rehabilitation Poststroke and Potential Contributions to Mobility Outcomes. Neurorehabilitation and neural repair. 2015;29(10):923-932.

4. Shum ST, Chiu JK, Tsang CP, et al. Predicting walking function of patients one month poststroke using modified Rivermead mobility index on admission. J Stroke Cerebrovasc Dis. 2014;23(8):2117-2121.

5. Kollen B, Kwakkel G, Lindeman E. Longitudinal robustness of variables predicting independent gait following severe middle cerebral artery stroke: a prospective cohort study. Clinical rehabilitation. 2006;20(3):262-268.

6. Hirano Y, Hayashi T, Nitta O, et al. Prediction of Independent Walking Ability for Severely Hemiplegic Stroke Patients at Discharge from a Rehabilitation Hospital. J Stroke Cerebrovasc Dis. 2016;25(8):1878-1881.

7. Bland MD, Sturmoski A, Whitson M, et al. Prediction of discharge walking ability from initial assessment in a stroke inpatient rehabilitation facility population. Archives of physical medicine and rehabilitation. 2012;93(8):1441-1447.

8. Louie DR, Eng JJ. Berg Balance Scale score at admission can predict walking suitable for community ambulation at discharge from inpatient stroke rehabilitation. Journal of rehabilitation medicine. 2018;50(1):37-44.

9. Veerbeek JM, Van Wegen EE, Harmeling-Van der Wel BC, Kwakkel G, Investigators E. Is accurate prediction of gait in nonambulatory stroke patients possible within 72 hours poststroke? The EPOS study. Neurorehabilitation and neural repair. 2011;25(3):268-274.

10. Smith MC, Barber PA, Stinear CM. The TWIST Algorithm Predicts Time to Walking Independently After Stroke. Neurorehabilitation and neural repair. 2017;31(10-11):955-964.

11. Kuys SS, Bew PG, Lynch MR, Morrison G, Brauer SG. Measures of activity limitation on admission to rehabilitation after stroke predict walking speed at discharge: an observational study. The Australian journal of physiotherapy. 2009;55(4):265-268.

12. Wee JY, Bagg SD, Palepu A. The Berg balance scale as a predictor of length of stay and discharge destination in an acute stroke rehabilitation setting. Archives of physical medicine and rehabilitation. 1999;80(4):448-452.

13. Rabadi $\mathrm{MH}$, Blau A. Admission ambulation velocity predicts length of stay and discharge disposition following stroke in an acute rehabilitation hospital. Neurorehabilitation and neural repair. 2005;19(1):20-26.

14. Scrivener K, Sherrington C, Schurr K. Amount of exercise in the first week after stroke predicts walking speed and unassisted walking. Neurorehabilitation and neural repair. 2012;26(8):932938.

15. Lang CE, Macdonald JR, Reisman DS, et al. Observation of amounts of movement practice provided during stroke rehabilitation. Archives of physical medicine and rehabilitation. 2009;90(10):1692-1698.

16. Moore JL, Roth EJ, Killian C, Hornby TG. Locomotor training improves daily stepping activity and gait efficiency in individuals poststroke who have reached a "plateau" in recovery. Stroke; $a$ journal of cerebral circulation. 2010;41(1):129-135. 
17. Zbogar D, Eng JJ, Miller WC, Krassioukov AV, Verrier MC. Movement repetitions in physical and occupational therapy during spinal cord injury rehabilitation. Spinal cord. 2017;55(2):172-179.

18. Scrivener K, Sherrington C, Schurr K. Exercise dose and mobility outcome in a comprehensive stroke unit: description and prediction from a prospective cohort study. Journal of rehabilitation medicine. 2012;44(10):824-829.

19. Zbogar D, Eng JJ, Noble JW, Miller WC, Krassioukov AV, Verrier MC. Cardiovascular Stress During Inpatient Spinal Cord Injury Rehabilitation. Archives of physical medicine and rehabilitation. 2017;98(12):2449-2456.

20. Prajapati SK, Mansfield A, Gage WH, Brooks D, Mcllroy WE. Cardiovascular responses associated with daily walking in subacute stroke. Stroke research and treatment. 2013;2013:612458.

21. Kuys S, Brauer S, Ada L. Routine physiotherapy does not induce a cardiorespiratory training effect post-stroke, regardless of walking ability. Physiotherapy research international : the journal for researchers and clinicians in physical therapy. 2006;11(4):219-227.

22. Mackay-Lyons MJ, Makrides L. Cardiovascular stress during a contemporary stroke rehabilitation program: is the intensity adequate to induce a training effect? Archives of physical medicine and rehabilitation. 2002;83(10):1378-1383.

23. Hornby TG, Straube DS, Kinnaird CR, et al. Importance of specificity, amount, and intensity of locomotor training to improve ambulatory function in patients poststroke. Topics in stroke rehabilitation. 2011;18(4):293-307.

24. Hornby TG, Moore JL, Lovell L, Roth EJ. Influence of skill and exercise training parameters on locomotor recovery during stroke rehabilitation. Current opinion in neurology. 2016;29(6):677683.

25. Hornby TG, Reisman DS, Ward IG, et al. Clinical Practice Guideline to Improve Locomotor Function Following Chronic Stroke, Incomplete Spinal Cord Injury, and Brain Injury. Journal of neurologic physical therapy : JNPT. 2020;44(1):49-100.

26. Hornby TG, Holleran CL, Hennessy PW, et al. Variable Intensive Early Walking Poststroke (VIEWS): A Randomized Controlled Trial. Neurorehabilitation and neural repair. 2016;30(5):440450.

27. Hornby TG, Henderson CE, Plawecki A, et al. Contributions of Stepping Intensity and Variability to Mobility in Individuals Poststroke. Stroke; a journal of cerebral circulation. 2019;50(9):24922499.

28. Hornby TG, Henderson CE, Holleran CL, Lovell L, Roth EJ, Jang JH. Stepwise Regression and Latent Profile Analyses of Locomotor Outcomes Poststroke. Stroke; a journal of cerebral circulation. 2020;51(10):3074-3082.

29. Moore JL, Nordvik JE, Erichsen A, et al. Implementation of High-Intensity Stepping Training During Inpatient Stroke Rehabilitation Improves Functional Outcomes. Stroke; a journal of cerebral circulation. 2020;51(2):563-570.

30. Kwakkel G, Kollen BJ. Predicting activities after stroke: what is clinically relevant? International journal of stroke : official journal of the International Stroke Society. 2013;8(1):25-32.

31. Charlson M, Szatrowski TP, Peterson J, Gold J. Validation of a combined comorbidity index. Journal of clinical epidemiology. 1994;47(11):1245-1251.

32. Holleran CL, Straube DD, Kinnaird CR, Leddy AL, Hornby TG. Feasibility and potential efficacy of high-intensity stepping training in variable contexts in subacute and chronic stroke. Neurorehabilitation and neural repair. 2014;28(7):643-651.

33. Borg G. Ratings of perceived exertion and heart rates during short-term cycle exercise and their use in a new cycling strength test. International journal of sports medicine. 1982;3(3):153-158.

34. Enright PL, Sherrill DL. Reference equations for the six-minute walk in healthy adults. Am J Respir Crit Care Med. 1998;158(5 Pt 1):1384-1387. 
35. Berg K, Wood-Dauphinee S, Williams JI. The Balance Scale: reliability assessment with elderly residents and patients with an acute stroke. Scand J Rehabil Med. 1995;27(1):27-36.

36. van Middendorp JJ, Hosman AJ, Donders AR, et al. A clinical prediction rule for ambulation outcomes after traumatic spinal cord injury: a longitudinal cohort study. Lancet. 2011;377(9770):1004-1010. 
429 Figure 1. Stepping activity at specific levels of locomotor recovery at discharge, including those 430 with $\operatorname{LoA} \leq 3, \mathrm{LoA}=4, \mathrm{LoA} \geq 5$ but $<0.4 \mathrm{~m} / \mathrm{s}, 0.4-0.8 \mathrm{~m} / \mathrm{s}$, and $\geq 0.8 \mathrm{~m} / \mathrm{s}$. The $\mathrm{x}$-axis is provided for 431 days of length of stay; average stepping in the first week is indicated at day 7 (end of first week) 432 at day 14 for the second week (end of second week) and at the day of discharge for the last week. 433 Error bars are standard deviations.

Figure 2. Logistic regressions for recovery of unassisted walking ability at admission (A) and using 1-week outcomes (B). Clinical approximations are provided with changes in the number of predictors and $\mathrm{x}$-axes at admission (C) and using 1-week outcome (D). 
Table 1. Participant demographics, clinical characteristics and training parameters [values as median (IQR) or count (\%)]. Duration post-stroke indicates days from stroke onset to admission to inpatient rehabilitation; $*$ indicates significantly differences at $\mathrm{p}<0.05$.

\section{$\underline{\text { Demographics }}$}

age (y)

gender: male

lesion location: right

left

bilateral

distribution: cortical

subcortical

subtentorial

multiple/unknown

lesion type: ischemic

hemorrhagic/unknown

duration post-stroke

Charlson Comorbidity Index

\section{Baseline assessments}

paretic leg strength

BBS

FIM-Walk

FIM-Transfers

FIM-Toilet transfers

\section{Training interventions}

length of stay

PT sessions

PT sessions/day

steps/day
$\mathrm{LOA} \leq 4$ at admission $(\mathbf{n}=\mathbf{2 5 7})$

$62(52-71)$

$158(61 \%)$

$109(42 \%)$

$103(40 \%)$

$45(18 \%)$

$113(44 \%)$

$48(19 \%)$

$46(18 \%)$

$47(19 \%)$

$164(64 \%)$

$93(36 \%)$

$13(8-26)$

$1(0-2)$

$$
\begin{gathered}
2.0(0.3-3.1) \\
5(3-8.5) \\
1(0-1) \\
3(2-4) \\
2(0-3)
\end{gathered}
$$

30 (24-38)

33 (26-42)

$1.1(0.9-1.2)$

1270 (553-2297)
$\underline{\mathrm{LOA}} \leq 4$ at discharge $(\mathrm{n}=116)$

$63(54-72)$

$73(63 \%)$

$50(43 \%)$

$44(38 \%)$

$22(19 \%)$

$51(44 \%)$

$24(21 \%)$

$21(18 \%)$

$19(17 \%)$

$72(62 \%)$

$44(38 \%)$

$17(10-36)^{*}$

$1(0-2)$

$$
\begin{gathered}
0.6(0.0-2.0)^{*} \\
3(1-5)^{*} \\
1(0-1) \\
1(0-2)^{*} \\
2(0-3)
\end{gathered}
$$

$$
\begin{gathered}
33(28-40)^{*} \\
34(27-42) \\
1.0(1.0-1.1) \\
538(310-873)
\end{gathered}
$$

$\underline{\mathrm{LOA}} \geq 5$ at

discharge $(n=141)$

$60(50-71)$

$85(60 \%)$

$59(42 \%)$

$59(42 \%)$

$23(16 \%)$

$62(45 \%)$

$24(17 \%)$

$25(18 \%)$

$28(20 \%)$

$92(65 \%)$

$49(35 \%)$

$12(7-21)^{*}$

$1(0-2)$

$$
\begin{gathered}
2.5(1.3-3.7)^{*} \\
6(4-18.5)^{*} \\
1(0-1) \\
3(2-4)^{*} \\
2(1-3)
\end{gathered}
$$

$29(23-38)^{*}$

$33(26-44)$

$1.1(1.0-1.3)$

$1979(1347-2835)$ 
Table 2. Outcomes at admission, week 1 and at discharge for primary balance and locomotor outcomes. (data presented as median [IQR $\}$ )

\section{$\underline{\mathrm{LOA}} \leq 4$ at admission $\quad \underline{\mathrm{LOA}} \leq 4$ at discharge $\quad \underline{\mathrm{LOA}} \geq 5$ at discharge}

\section{$\underline{B B S}$}

admit

week 1

discharge

$\underline{10 M W T}$

admit

week 1

discharge

6MWT

admit

week 1

discharge

6MWT LoA

admit

week 1

discharge

\section{$\underline{(\mathbf{n}=257)}$}

5 (3-9)

7 (4-22)

$26(8-43)$

$0(0-0.12)$

$0.08(0-0.33)$

$0.31(0.06-0.71)$

7 (0-23)

$21(5-92)$

96 (23-236)

$2(1-3)$

$3(2-4)$

5 (3-5) $\underline{(n=116)}$

$\underline{(n=141)}$

$3(1-5)$

6 (4-19)

$4(3-6)$

20 (7-33)

$6(4-17)$

41 (31-47)

$0(0-0)$

$0.08(0-0.23)$

$0(0-0.07)$

$0.25(0.07-0.51)$

$0.07(0-0.18)$

$1(0-9)$

$8(0-20)$

$20(0-53)$

1 (1-2)

2 (1-2)

3 (1-4)
$16(6-44)$

0.63 (0.38-0.95)

75 (20-168)

213 (117-317)

$3(2-4)$

$4(3-5)$

$5(5-5)$ 
Table 3. Correlation analyses of demographics, baseline clinical characteristics and training parameters on primary discharge outcomes. $* \mathrm{p}<0.05, * * \mathrm{p}<0.01$

\author{
Demographics \\ age \\ duration post-stroke \\ Baseline characteristics \\ paretic strength \\ admit BBS \\ admit 10MWT \\ admit 6MWT \\ admit 6MWT LoA \\ Training characteristics \\ length of stay \\ PT sessions \\ steps/day
}

BBS

$\begin{array}{llll}-.20 & -.16 & -.15 & -.12 \\ -.24^{*} & -.24^{*} & -.25^{*} & -.26^{*} \\ & & & \\ .43^{*} & .46^{*} & .46^{*} & .45^{*} \\ .60^{* *} & .59^{*} & .57^{*} & .60^{*} \\ .49^{* *} & .58^{*} & .55^{*} & .46^{*} \\ .55^{* *} & .60^{*} & .61^{*} & .55^{*} \\ .51^{* *} & .54^{*} & .57^{*} & .54^{*} \\ & & & \\ -.12 & -.18 & -.17 & -.15 \\ .05 & .00 & .02 & -.01 \\ .75^{*} & .81^{*} & .83^{*} & .74^{*}\end{array}$


Table 4. Cut-off thresholds (area under the curve) for ROC analyses for each locomotor threshold for functional mobility.

\begin{tabular}{|c|c|c|c|c|}
\hline \multicolumn{2}{|c|}{$\underset{\text { Demographics/bassline characteristics }}{\underline{\mathrm{LoA} \geq 4}}$} & $\underline{\operatorname{LoA}} \geq \underline{5}$ & $\geq 0.4 \mathrm{~m} / \mathrm{s}$ & $\geqq 0.8 \mathrm{~m} / \mathrm{s}$ \\
\hline age (y) & -- & -- & $\begin{array}{c}58.8 \\
57(0.50-0.65)\end{array}$ & $\begin{array}{c}58.8 \\
.64(0.55-0.72)\end{array}$ \\
\hline duration & $\begin{array}{c}14.5 \\
0.66(0.59-0.73)\end{array}$ & $\begin{array}{l}10.5 \\
63(0.56-0.70)\end{array}$ & $\begin{array}{c}10.5 \\
0.61(0.54-0.68)\end{array}$ & $\begin{array}{c}29.5 \\
0.60(0.52-0.68)\end{array}$ \\
\hline stren & $\begin{array}{c}2.3 \\
0.73(0.67-0.8\end{array}$ & $\begin{array}{c}1.8 \\
0.74(0.68-0.80)\end{array}$ & $\begin{array}{c}1.8 \\
0.74(0.68-0.81)\end{array}$ & $\begin{array}{c}1.8 \\
0.77(0.70-0.84)\end{array}$ \\
\hline Outco & & & & \\
\hline BBS & $0.82(0.7$ & $\begin{array}{c}5.5 \\
0.79(0.73-0.85)\end{array}$ & $\begin{array}{c}5.5 \\
0.80(0.74-0.85)\end{array}$ & $\begin{array}{c}5.5 \\
0.76(0.69-0.84)\end{array}$ \\
\hline 10MWT (m/s & $\begin{array}{r}0.01 \\
0.75(0.69\end{array}$ & $\begin{array}{c}0.03 \\
0.72(0.66-0.78)\end{array}$ & $\begin{array}{c}0.08 \\
0.75(0.68-0.81)\end{array}$ & $\begin{array}{c}0.19 \\
0.73(0.65-0.82)\end{array}$ \\
\hline 6MWT (m) & $\begin{array}{c}2 \\
0.83(0.78\end{array}$ & $\begin{array}{c}6 \\
0.77(0.72-0.83)\end{array}$ & $\begin{array}{c}18 \\
0.76(0.70-0.82)\end{array}$ & $\begin{array}{c}18 \\
0.77(0.69-0.85)\end{array}$ \\
\hline $6 \mathrm{MW}$ & $\begin{array}{c}2.5 \\
0.83(0.78-\mathrm{C}\end{array}$ & $\begin{array}{c}2.5 \\
0.78(0.73-0.84)\end{array}$ & $\begin{array}{c}2.5 \\
0.74(0.68-0.81)\end{array}$ & $\begin{array}{c}2.5 \\
0.74(0.66-0.82)\end{array}$ \\
\hline anc & & & & \\
\hline BBS & $\begin{array}{c}5.5 \\
0.88(0.84-0 .\end{array}$ & $\begin{array}{c}9.5 \\
0.86(0.81-0.90)\end{array}$ & $\begin{array}{c}9.5 \\
0.86(0.81-0.91)\end{array}$ & $\begin{array}{c}16.5 \\
0.85(0.79-0.91)\end{array}$ \\
\hline 10MWT $(\mathrm{m} / \mathrm{s}$ & $\begin{array}{c}0.14 \\
0.84(0.79-0.89)\end{array}$ & $\begin{array}{c}0.14 \\
0.84(0.79-0.89)\end{array}$ & $\begin{array}{c}0.14 \\
0.86(0.81-0.92)\end{array}$ & $\begin{array}{c}0.21 \\
0.86(0.79-0.92)\end{array}$ \\
\hline 6MWT (n & $\begin{array}{c}31 \\
0.88(0.83-0.92)\end{array}$ & $\begin{array}{c}36 \\
0.85(0.80-0.89)\end{array}$ & $\begin{array}{c}49 \\
0.86(0.81-0.91)\end{array}$ & $\begin{array}{c}90 \\
0.87(0.81-0.93)\end{array}$ \\
\hline 6MWT Lo & $\begin{array}{c}2.5 \\
0.90(0.86-0.93)\end{array}$ & $\begin{array}{c}2.5 \\
0.87(0.83-0.91)\end{array}$ & $\begin{array}{c}3.5 \\
0.83(0.77-0.88)\end{array}$ & $\begin{array}{c}3.5 \\
0.79(0.73-0.86)\end{array}$ \\
\hline
\end{tabular}




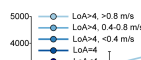

?

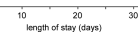




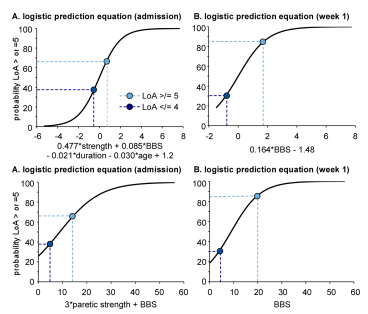

